\title{
Minocyclin bei Akne - autoimmunerkrankungsartiges Syndrom als Nebenwirkung
}

Frage:

Im 'Arznei-Telegramm' 2/96 (Berlin) wird auf Immunerkrankungen (LE-ähnliches Syndrom, letal verlaufende Hepatitiden) unter der systemischen Gabe von Minocyclin (Klinomycin) bei Akne papulopustulosa hingewiesen. Die norwegische Gesundheitsbehörde habe 1989 die Zulassung von Minocyclin wegen dieses Nebenwirkungsprofiles versagt. Empfohlen wird als besser verträgliche Alternative $50 \mathrm{mg}$ Doxycyclin/d. Sind Ihnen derartige Nebenwirkungen bekannt und teilen Sie die genannten Bedenken?

\section{Antwort:}

Die in der „Anfrage aus der Praxis“ genannte Quelle referiert im wesentlichen eine kürzlich im British Mecial Journal erschienene Arbeit von Gough et al. [2]. In dieser Publikation werden bei mit Minocyclin (Klinomycin $\left.{ }^{\circledR}\right)$, einem Tetrazyklin, behandelten Patienten beobachtete, bekannte, seltene Nebenwirkungen beschrieben. Es handelt sich hierbei um ein dem Lupus erythematodes ähnliches durch Arthralgien gekennzeichnetes Syndrom, eine histologisch als chronisch aktiv charakterisierte Hepatitis, sowie ein in einigen Fällen gemeinsames Auftreten der beiden Erscheinungen. Als Hinweis auf einen nicht nur zeitlichen Zusammenhang mit der Anwendung des Arzneimittels wird die Reversibilität der Krankheitserscheinungen nach Absetzen und in einigen Fällen beobachtetes Wiederauftreten nach Reexposition angeführt. Die Autoren diskutieren jedoch gleichfalls, daß spontan auftretende Autoimmunhepatitiden hinsichtlich Geschlechts- und Altersverteilung mit den im Zusammenhang mit der Anwendung von Minocyclin (Klino$\operatorname{mycin}^{\circledR}$ ) beobachteten konkordant sind, und daß auch die Akne selbst eine Arthritis auslösen kann und häufig mit einer autoimmuninduzierten $\mathrm{He}$ patitis vergesellschaftet ist.

Diese jetzt aus Großbritanien berichteten seltenen Nebenwirkungen sind für Vertreter der Substanzklasse der Tetrazykline seit langem beschrieben. Zur praktischen Bedeutung der von Gough et al. zusammengestellten Befunde nimmt Cunliffe, einer der erfahrensten internationalen Spezialisten auf dem Gebiet der Aknetherapie, in einem Brief an die Herausgeber des BMJ Stellung [1]. Cunliffe überblickt 25000 in einem Zeitraum von 26 Jahren wegen Akne behandelte Patienten, von denen viele $(>85 \%)$ Minocyclin (Klinomycin $\left.{ }^{\circledR}\right)$ erhalten haben. In diesem Kollektiv entwickelten 3 Patienten eine reversible Hepatitis und ein Patient ein dem Lupus erythematodes ähnliches Syn- drom mit persistierender Arthritis. Von Interesse erscheint im Hinblick auf eine Nutzen-Risiko-Bewertung, daß sich im überblickten Zeitraum 5 Patienten im Rahmen einer mit der Akne assoziierten Depression suizidierten und 51 weitere wegen einer Medikamentenüberdosierung hospitalisiert wurden. Demgegenüber trat kein Todesfall bei Patienten auf, die Minocyclin (Klinomycin ${ }^{\circledR}$ ) einnahmen.

Zwischenzeitlich hat auch das britische Committe on the Safety of Medicines (CMS) mitgeteilt, es sehe keine Notwendigkeit für eine Änderung des Verschreibungsverhaltens.

Nach über 20jähriger Anwendungserfahrung bleibt Minocyclin (Klinomycin ${ }^{\circledR}$ ) durch seine Wirksamkeit und Verträglichkeit einer der Stützpfeiler der Aknetherapie.

Auf die Möglichkeit des Auftretens der beschriebenen und in der Regel reversiblen Nebenwirkungen und ihre Manifestationsformen hingewiesen $\mathrm{zu}$ haben, ist sicherlich ein Verdienst der Autoren, da hierdurch erneut die Notwendigkeit ins Bewußtsein gerückt wird, auf entsprechende Symptome bei jedem Patientenkontakt zu achten.

\section{Literatur}

1. Cunliffe WJ (1996) Doctors should not change the way they prescribe for acne. BMJ 312: 1101

2. Gough A, Chapman S, Wagstaff K, Emery $P$, Elias E (1996) Minocycline induced autoimmune hepatitis and systemic lupus erythematosus-like syndrome. BMJ 312: 169-172

Dr. W. Jagusch

Wyeth Gruppe

Postfach 8806

D-48136 Münster 